\title{
The Role of Intracarotid Cold Saline Infusion on a Theoretical Brain Model Incorporating the Circle of Willis and Cerebral Venous Return
}

\author{
Matthew A. Neimark, Angelos-Aristeidis Konstas, Jae H. Choi, Andrew F. Laine, and John Pile- \\ Spellman
}

\begin{abstract}
This study describes a theoretical model of brain cooling by intracarotid cold saline infusion which takes into account redistribution of cold perfusate through the circle of Willis (CoW) and cold venous return (VR) from the head. This model is developed in spherical coordinates on a four tissue layer hemispherical geometrical configuration.

Temperature evolution is modeled according to the Pennes bioheat transfer equation. Simulations were run over a 1 hour period and $30 \mathrm{ml} / \mathrm{min}$ of freezing cold saline with the baseline model (no VR, no CoW), VR model (without CoW), and CoW model (with VR). The VR model demonstrates continuing temperature drop in the treatment region of the brain not observed in the baseline model and its final mean ipsilateral anterior temperature was approximately $31^{\circ} \mathrm{C}$. The temperature effect in the $\mathrm{CoW}$ model was present but less robust in the ipsilateral anterior region, as final temperature was $32{ }^{\circ} \mathrm{C}$. However, cooling was also achieved in contralateral and posterior brain regions. This model continues to demonstrate the feasibility of intracarotid cold saline infusion for ischemic stroke therapy.
\end{abstract}

\section{INTRODUCTION}

$\mathrm{H}$ ypothermia is a neuroprotective treatment approach proven to be beneficial in brain ischemia caused by cardiac arrest [1]. It has also shown positive results for acute ischemic stroke in animal models [2] and small clinical series [3]. To administer hypothermia, practitioners have traditionally used whole-body cooling methods such as inferior vena cava cooling catheters [4] or surface cooling [5]. These systemic cooling methods take a long period of time to achieve therapeutic temperatures, and involve side effects, such as arrhythmias, coagulapathies, and infections [3]. Attempted selective brain cooling methods have included ice packs and cooling helmets [6], but these methods have been determined to be largely ineffective [7, 8].

A recent study explores a selective cooling technique for ischemic stroke through administration of intracarotid cold saline [9]. In this study, heat transfer in the brain was mathematically modeled, and the expected temperature response was determined for a range of input flow rates.

Manuscript received March 29, 2007.

M. A. Neimark and A. F. Laine are with the Department of Biomedical Engineering, Columbia University, New York, NY 10027 USA (phone: 212-854-5996; fax: 212-854-5995; e-mail: man2003@ columbia.edu).

A. A. Konstas, J. H. Choi, and J. Pile-Spellman are with the Department of Radiology, Columbia University, New York, NY 10032 USA (e-mail: jp59@ columbia.edu).
From this study, it was determined that selective brain cooling to $32^{\circ} \mathrm{C}$ could be attained using ice-cold saline at a flow rate of $30 \mathrm{ml} / \mathrm{min}$. Furthermore, these therapeutic levels of hypothermia could be achieved within 10 minutes, a substantial methodical and technological improvement over systemic strategies (eg. inferior vena cava cooling catheters [4].)

This brain cooling model employed a number of assumptions and simplifications. One such simplification was that infused cold saline only affected a particular perfusion territory corresponding to the ipsilateral anterior and middle cerebral artery. In reality, however, the presence of the circle of Willis (CoW) enables cooled saline, infused through the internal carotid artery, to be distributed to the opposite side of the brain through the anterior communicating artery, or to the posterior perfusion territory through the ipsilateral posterior communicating artery (see Fig. 1).

Another assumption made in the previous model is that core temperature, which determines the temperature of blood feeding the brain in the model, was constant at normal body temperature $\left(37^{\circ} \mathrm{C}\right)$. However, when the cold saline mixes with arterial blood to cool the brain, this cooled blood returns from the brain (venous return, VR) to cool the core temperature too.

In this present study, the effects of the CoW and VR are implemented into a new enhanced model which explores the effect of intracarotid cold saline infusion on the temperature response in the human brain. With VR, the temperature response is expected to be both quicker and more pronounced. The questions regarding VR are 1) the magnitude of this enhanced cooling and response and 2) whether body temperature will be sustained near normal, or whether there will be a significant effect of systemic hypothermia from VR.

Similarly, this study will examine the effect of redistribution of cold saline throughout the brain as a result of the CoW. This should both blunt the temperature response in the ipsilateral anterior territory, and introduce cooling in the anterior contralateral and posterior ipsilateral territories. The questions regarding the $\mathrm{CoW}$ relate to 1) the degree of blunting of the cooling effect in the anterior ipsilateral territory due to redistribution of cold saline and 2) the amount of cooling that will be introduced into these other territories. 


\section{METHODS}

\section{A. Heat Transfer Model}

Heat transfer is simulated in a three dimensional hemispherical mathematical model, developed in spherical coordinates, and consisting of four separate tissue layers: white matter, gray matter, bone, and skin. Heat transfer develops over space and time according to the Pennes bioheat equation [10]:

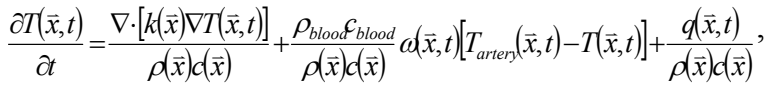

where $T$ is tissue temperature, $k$ is the tissue thermal conductivity, $\rho$ is density, $c$ is heat capacity, $\omega$ blood perfusion, $q$ is tissue metabolism (also corrected in the brain for temperature), $t$ is the time point, and $\vec{x}$ is the spatial location. The subscript "blood" signifies blood density and heat capacity while the subscript "artery" signifies arterial temperature. All blood has core temperature except for the ipsilateral ICA whose temperature is

$$
T_{I C A}=\frac{\rho_{\text {blood }} c_{\text {blood }} \text { ICA Blood Flow } \cdot T_{\text {core }}+\rho_{\text {saline }} c_{\text {saline }} \text { Saline Flow } \cdot T_{\text {saline }}}{\rho_{\text {blood }} c_{\text {blood }} \text { ICA Blood Flow }+\rho_{\text {saline }} c_{\text {saline }} \text { Saline Flow }}
$$

For brain tissue, metabolism is a function of temperature according to $q=q_{0} \cdot 3.0^{0.084(T-37)}$ (3) and perfusion is a function of temperature and hematocrit according $\omega=\omega_{0} \cdot 3.0^{0.084(T-37)}\left(1-2.2 \Delta_{H C T}\right) \quad$ (4) [9]. Further details of the formulation of the heat transfer model and parameter values have been previously presented [9].

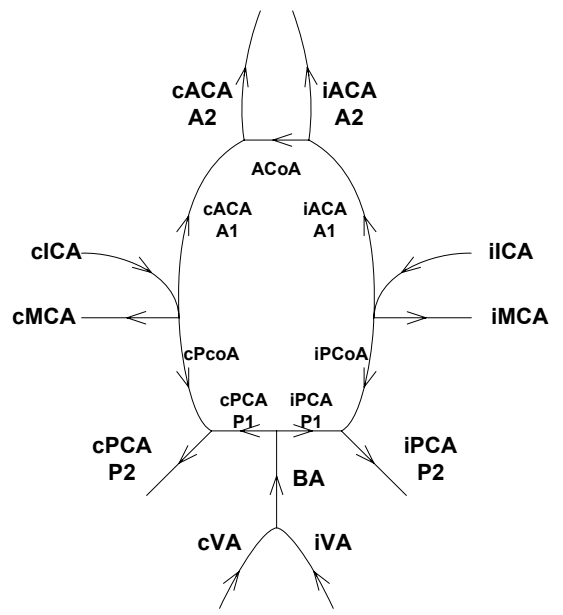

Fig. 1. Diagram of circle of Willis (CoW) showing normative flow patterns. Source vessels include the internal carotid arteries (ICA) and vertebral arteries (VA) which join to form the basilar artery (BA). CoW vessels include the anterior communicating artery (AcoA), A1 branch of the anterior cerebral artery (ACA), posterior communicating artery (PCoA), and P1 branch of the posterior cerebral arteries. CoW efferent vessels, which perfuse brain tissue, include the A2 branches of the ACA and PCA, and the middle cerebral artery MCA. Prefix "i" implies ipsilateral while "c" implies contralateral.

\section{B. Venous Return}

Since blood and brain tissues achieve thermal equilibrium [11], VR temperatures were calculated by integrating the product of the brain temperature and blood perfusion over the entire brain model as follows:

$$
T_{\text {venous }}=\iiint_{\text {Brain Model }} \omega(\vec{r}) T(\vec{r}) d \vec{r} / \iiint_{\text {Brain Model }} \omega(\vec{r}) d \vec{r}
$$

$T_{\text {venous }}$ is the average VR blood temperature which has been cooled by ICSI.

If this venous blood is assumed to equilibrate with the core temperature, an expression to evaluate the evolving core temperature is:

$$
\frac{d T_{\text {core }}}{d t}=\frac{\rho_{\text {blood }} c_{\text {blood }}\left(T_{\text {venous }}-T_{\text {core }}\right) \iiint_{\text {brain } \bmod e l} \omega(\vec{r}) d \vec{r}}{m_{\text {body }} c_{\text {body }}}
$$

where $\rho_{\text {blood }}$ is the density of blood and $T_{\text {core }}$ is the core temperature.

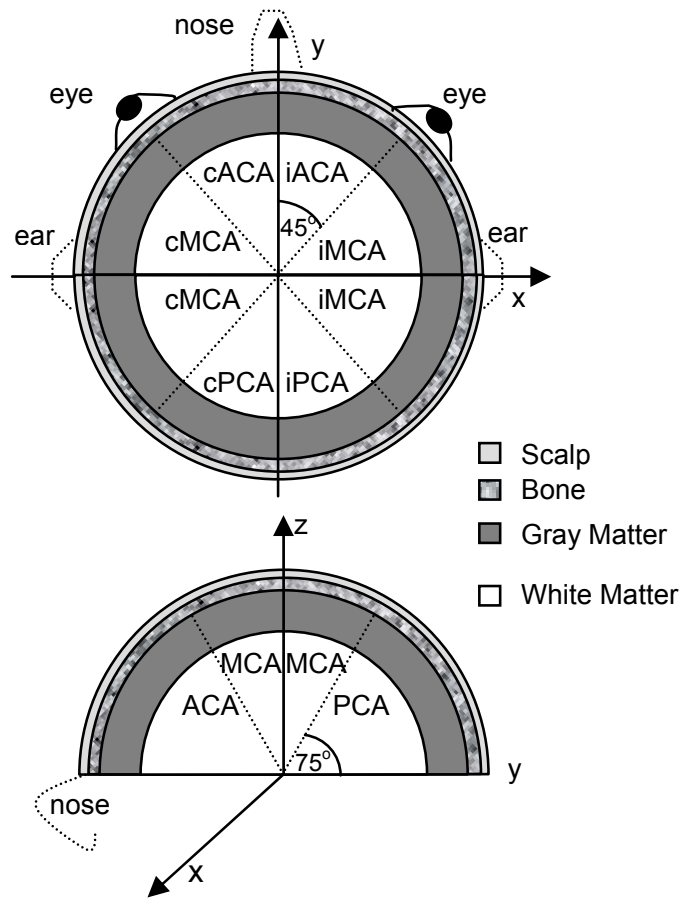

Fig. 2. Geometry of brain perfusion model (top - axial view and bottom paramedian view) showing four tissue layers and demarcation of perfusion territories.

\section{Circle of Willis}

Modelling of the CoW was accomplished according to a linear model [13]. According to Poiseuille's law, the flow in a vessel is determined as follows: $F=G\left(P_{2}-P_{1}\right)$ (7) where $F$ is the flow, $P_{2}$ and $P_{1}$ are the pressures at the two ends of the vessels and $G$ is the flow conductance. $G$ itself is determined as follows: $G=\frac{\pi d^{4}}{128 \eta_{\text {blood }} L}$ (8) where $\eta_{\text {blood }}$ is blood viscosity, $L$ is vessel length, and $d$ is vessel diameter .

The hemispheric model was divided up into six perfusion territories corresponding to each of the cerebral arteries. Fig. 2 shows axial and paramedian diagrams describing the geometry of the perfusion territories. In general, the MCA territories take up the middle $90^{\circ}$ of the axial domain at the 
base of the brain (i.e. $|\phi|<45^{\circ}$ for the left MCA and $\left|\phi+180^{\circ}\right|<45^{\circ}$ for the right MCA). The ACA and PCA territories make up the remaining anterior and posterior domain as shown in Fig 2 (top). However, the MCA territories also encompass all areas where $|\theta|<15^{\circ}$ as shown in Fig. 2 (bottom).

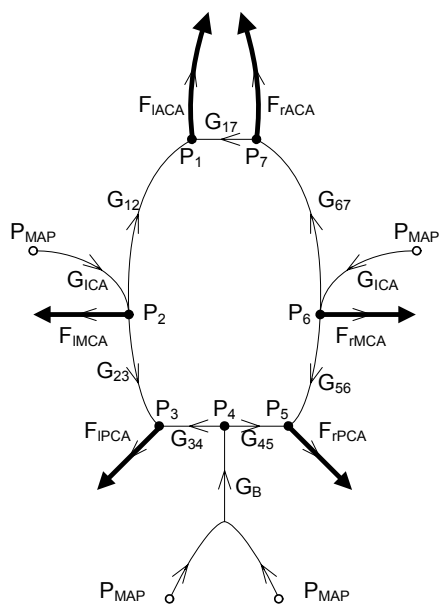

Fig. 3. Circuit model of CoW model. Vessels in bold reperesent flow sources determined by integrating flow cerebral flow values in corresponding perfusion regions (see Fig. 2). Values $P_{i}$ represent pressure values at vessel junction points within the CoW. Values $G_{m n}$ represent vascular conductance values of corresponding vessels between vessel junction point $\mathrm{m}$ and $\mathrm{n}$. Open circles represent pressures at the origin of the source vessels which are clamped to mean arterial pressure.

Perfusion $(\omega)$ values in the brain are still assumed to be determined solely by temperature and hematocrit according to (4). Cerebral perfusion pressure is assumed not to play a role in cerebral blood flow because it is assumed autoregulation is still intact. Flow rates through the cerebral arteries can then be determined by integrating the $\omega$ values in the corresponding perfusion territories in Fig. 2 according to $F=\iiint \int_{\text {Territory }} \omega d V(9)$.

Blood flows in the rest of the CoW and supply vessels (i.e. ICAs and BA) will then be determined by Kirchoff's circuit laws (i.e the sum of incoming flows towards an incoming arterial junction is 0 and the sum of pressure changes in a closed arterial circuit is 0 ). In this manner, the cerebral arteries act as flow sources (hydrodynamic equivalent of current sources in an electric circuit, and pressures of the origins of the ICAs and VAs are clamped to mean arterial pressure. The pressure values $P_{i}$ can be .determined according to the matrix equation $\mathbf{G P}=\mathbf{F} \quad$ (10) where $\mathbf{G}$ is a matrix of conductances whose off diagonal elements $\mathbf{G}_{m n}$ is the conductance between node $m$ and $n$ and whose diagonal elements $\mathbf{G}_{m m}$ are the negative of the sum of the conductances of all vessels which join at node $m . \quad \mathbf{P}$ is a column vector of pressures $P_{i}$ corresponding to node $i$. $\quad \mathbf{F}$ is a column vector of flows $F_{i}$ corresponding to node $i$ and equaling $F_{e v}-M A P \cdot G_{s v}$ (11) where $F_{e v}$ is the flow of any efferent vessel at node $i, M A P$ is the mean arterial pressure, and $G_{s v}$ is the conductance of any afferent vessel at node $i$.

The conductance values in this matrix were calculated according to (8). After solving for $\mathbf{P}$, which contains all the $\mathrm{P}_{\mathrm{i}}$ values of the CoW, flow values in all the CoW and source vessels are calculated according to (9). This includes the ICA's, whose ipsilateral content includes the cold saline and blood perfusate and whose contralateral content includes core temperature blood, and the vertebral arteries, whose content consists of core temperature blood. The combination of these flows in the CoW will permit calculation of temperature and hematocrit in the cerebral vessels.

Once flows throughout the CoW model are known, temperature and hematocrit values can be determined for all vessels in the model. For every junction of vessels, one can determine via (7) which vessels have flows that enter into the junction, and which vessels have flows that emerge from the junction. The temperatures and hematocrits of the flows of the vessels enter into the junction mix according to

$$
T=\frac{\sum_{i=1}^{n} T_{i} F_{i} \rho_{i} c_{i}}{\sum_{i=1}^{n} F_{i} \rho_{i} c_{i}} \text { (12) and } H C T=\frac{\sum_{i=1}^{n} H C T_{i} F_{i}}{\sum_{i=1}^{n} F_{i}}
$$

where $n$ is the number of inflowing vessels and the subscripted values correspond to each of the inflowing vessels. The flows of the vessels emerging from the junction all have temperature $T$ and hematocrit $H C T$. All the temperatures in the vessels of the CoW can be determined using the temperatures of the source vessels, acquired from (2) and (5). Ultimately, the temperatures of the cerebral arteries are calculated and used as the arterial temperatures in (1) to propogate the brain temperature.

\section{RESULtS}

Three simulations were performed with a flow rate of 30 $\mathrm{ml} / \mathrm{min}$ of freezing cold saline over a course of 1 hour: 1) no VR or CoW, 2) VR but no CoW, 3) VR and CoW. Temporal and spatial temperature results of these simulations are shown in Fig. 4. The model without VR demonstrates mean ipsilateral anterior temperature (IAT), rapidly decreasing to a therapeutic level of approximately 32 ${ }^{\circ} \mathrm{C}$ and then slowly increasing after 20 minutes of infusion as previously observed due to increased hemodilution [9]. The model with VR shows mean IAT continuing to decrease after this point. The CoW model also shows mean IAT rapidly decreasing, but reaching a slowing phase of temperature diminishment at a higher temperature than the non CoW simulations. In any case, mean IAT temperature for the $\mathrm{CoW}$ reaches approximately $32^{\circ} \mathrm{C}$ after an hour. The modeling also showed that with VR (also in the CoW model), the body temperature cooled $1{ }^{\circ} \mathrm{C}$ over 1 hour.

Spatial results (Fig. 4) demonstrate therapeutic and uniform cooling in the IAT for all three models. For the VR model, cooling in the non-IAT regions also occurred due to body temperature lowering. In the CoW model, there was 
less cooling in the IAT than in the VR model. However, moderate cooling $\left(35^{\circ} \mathrm{C}\right)$ took place in the anterior contralateral and posterior ipsilateral territories.
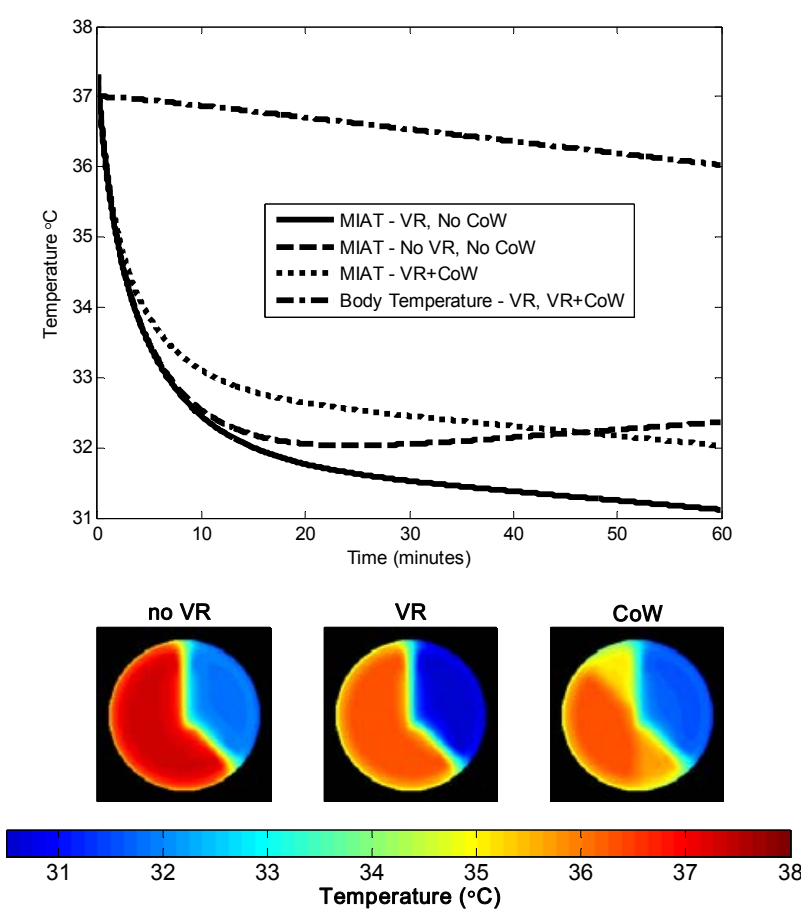

Fig. 4. Temporal (top) and spatial results (bottom) of freezing cold saline temperature infusion $(30 \mathrm{ml} / \mathrm{min})$. Temporal data sampled body temperature and mean anterior ipsilateral territory (MIAT) temperature. The ipsilateral anterior territory is the region perfused by the ipsilateral MCA and ACA vessels. Spatial results show axial brain slice at halfway point between base of brain and top of the head after 60 minutes of freezing cold saline infusion.

Cold perfusate is able to reach these territories via the anterior communicating and ipsilateral posterior communicating arteries. Fig. 5 demonstrates the evolution of flow rates in these vessels over the course of the simulation. The anterior communicating artery, which immediately after infusion has a slight contralateral to ipsilateral flow of $4 \mathrm{ml} / \mathrm{min}$ (due to hemodilution) quickly reverses and by 10 minutes of infusion delivers $20 \mathrm{ml} / \mathrm{min}$ of cold perfusate to the contralateral territory. The ipsilateral posterior communicating artery similarly doubles from 5 to $10 \mathrm{ml} / \mathrm{min}$.

\section{CONCLUSIONS}

This study demonstrates the effect of the CoW and VR in a model of brain cooling by intracarotid freezing cold saline. As expected, VR contributed to the cooling effect, eliminating the temperature rise due to hemodilution in the non-VR model and cooling the affected region of brain down to approximately $31{ }^{\circ} \mathrm{C}$. Body temperature decreased to $36{ }^{\circ} \mathrm{C}$, which was also within the predicted range, and avoids most systemic complications [9]. With the CoW model, the temperature within the treated region was slightly higher than in the non-CoW model, but still well within therapeutic levels. In addition, moderate cooling was introduced to regions in the contralateral hemisphere and posterior perfusion regions. This model demonstrates the feasibility of intracarotid cold saline infusion for ischemic stroke therapy because of its speed and avoidance of systemic hypothermia.

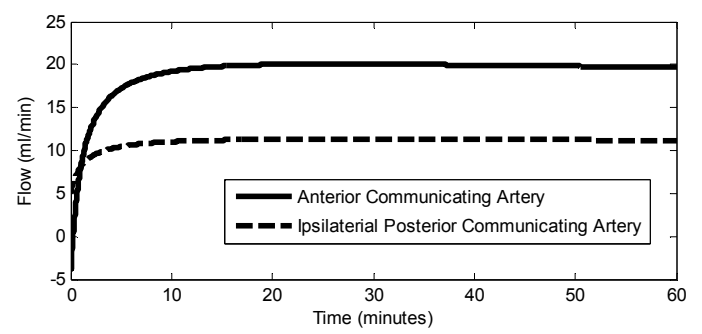

Fig. 5. Flow rates of anterior communicating artery. Positive flow directions are shown in Fig. 1

\section{REFERENCES}

[1] M. Holzer, S. A. Bernard, S. Hachimi-Idrissi, R. O. Roine, F. Sterz, M. Mullner, and A. on behalf of the Collaborative Group on Induced Hypothermia for Neuroprotection After Cardiac, "Hypothermia for neuroprotection after cardiac arrest: systematic review and individual patient data meta-analysis.," Critical Care Medicine, vol. 33, pp. 414$8,2005$.

[2] A. A. Konstas, J. H. Choi, and J. Pile-Spellman, "Neuroprotection for ischemic stroke using hypothermia," Neurocritical Care, vol. 4, pp. 168-78, 2006.

[3] S. Schwab, S. Schwarz, M. Spranger, E. Keller, M. Bertram, and W. Hacke, "Moderate hypothermia in the treatment of patients with severe middle cerebral artery infarction.," Stroke, vol. 29, pp. 2461-6, 1998.

[4] M. A. De Georgia, D. W. Krieger, A. Abou-Chebl, T. G. Devlin, M. Jauss, S. M. Davis, W. J. Koroshetz, G. Rordorf, and S. Warach, "Cooling for Acute Ischemic Brain Damage (COOL AID): a feasibility trial of endovascular cooling," Neurology, vol. 63, pp. 3127, 2004.

[5] D. W. Krieger, M. A. De Georgia, A. Abou-Chebl, J. C. Andrefsky, C. A. Sila, I. L. Katzan, M. R. Mayberg, and A. J. Furlan, "Cooling for acute ischemic brain damage (cool aid): an open pilot study of induced hypothermia in acute ischemic stroke," Stroke, vol. 32, pp. 1847-54, 2001.

[6] B. A. Harris and P. J. D. Andrews, "Direct brain cooling," in Therapeutic Hypothermia, S. A. Mayer and D. I. Sessler, Eds. New York: Marcel Dekker, 2005, pp. 323-86.

[7] R. J. Corbett and A. R. Laptook, "Failure of localized head cooling to reduce brain temperature in adult humans," Neuroreport, vol. 9, pp. 2721-5, 1998

[8] P. Mellergard, "Changes in human intracerebral temperature in response to different methods of brain cooling," Neurosurgery, vol. 31, pp. 671-7; discussion 677, 1992.

[9] M. A. Neimark, A. A. Konstas, A. F. Laine, and J. Pile-Spellman, "Theoretical model of selective cooling using intracarotid cold saline infusion in the human brain," Journal of Applied Physiology, vol. [Epub ahead of print], December, 20062006.

[10] H. H. Pennes, "Analysis of tissue and arterial blood temperature in the resting human forearm," Journal of Applied Physiology, vol. 1, pp. 93$122,1948$.

[11] J. W. Baish, "Microvascular heat transfer," in The Biomedical Engineering Handbook, 2nd Ed. vol. 2, J. D. Bronzio and D. Joseph, Eds. Boca Raton, FL: CRC, 2000, pp. 98-1 - 98-14.

[12] D. Fiala, K. J. Lomas, and M. Stohrer, "A computer model of human thermoregulation for a wide range of environmental conditions: the passive system," Journal of Applied Physiology, vol. 87, pp. 1957-72, 1999.

[13] B. Hillen, B. A. Drinkenburg, H. W. Hoogstraten, L. Post, B. Hillen, B. A. Drinkenburg, H. W. Hoogstraten, and L. Post, "Analysis of flow and vascular resistance in a model of the circle of Willis.," Journal of Biomechanics, vol. 21, pp. 807-14, 1988. 